\title{
Restless legs syndrome and health-related quality of life in adults with multiple sclerosis
}

\author{
Katie L. J. Cederberg ${ }^{1}$ (D) | Brenda Jeng ${ }^{1}$ | Jeffer E. Sasaki ${ }^{2}$ | Tiffany J. Braley ${ }^{3}$ | Arthur \\ S. Walters ${ }^{4}$ | Robert W. Motl ${ }^{1}$
}

\author{
${ }^{1}$ Department of Physical Therapy, University \\ of Alabama at Birmingham, Birmingham, \\ Alabama \\ ${ }^{2}$ Department of Sports \\ Sciences, Universidade Federal do Triângulo \\ Mineiro, Bairro Tutunas, Uberabam, Brazil \\ ${ }^{3}$ Department of Neurology, University of \\ Michigan, Ann Arbor, Michigan \\ ${ }^{4}$ Department of Neurology, Vanderbilt \\ University, Nashville, Tennessee

\section{Correspondence} \\ Katie L. J. Cederberg, 1720 2nd Avenue \\ South, Birmingham, AL, USA. \\ Email: kcederb@uab.edu \\ Funding information \\ This work was supported, in part, by a pilot \\ grant from the National Multiple Sclerosis \\ Society (PP 1412) and a mentor-based \\ post-doctoral fellowship from the National \\ Multiple Sclerosis Society (MB 0011). The \\ funding source had no involvement in (a) the \\ study design, (b) data collection, analysis \\ or interpretation, (c) writing of the report, \\ or the decision to submit the article for \\ publication
}

\begin{abstract}
Restless legs syndrome (RLS) is a sleep disorder that may exacerbate many of the symptoms and consequences of multiple sclerosis (MS), and may have further implications for health-related quality of life (HRQOL). The present study examined the relationships among RLS, symptoms and HRQOL in people with MS. Participants with MS ( $n=275$ ) completed the Cambridge-Hopkins Restless Legs Syndrome Questionnaire, the International Restless Legs Syndrome Study Group Scale, the Multiple Sclerosis Impact Scale, the Pittsburgh Sleep Quality Index, the Fatigue Severity Scale, the Hospital Anxiety and Depression Scale and the Patient Determined Disease Steps. There were 74 (26.9\%) persons with MS who had RLS (MS + RLS). The MS + RLS group reported worse physical and psychological HRQOL ( $p=0.020$ and $p=0.017$, respectively) and greater perceived fatigue ( $p=0.006)$ and anxiety symptoms $(p=0.042)$ than the MS-only group. Within the MS + RLS group, RLS severity was associated with physical ( $r=0.43$ ) and psychological ( $r=0.46)$ HRQOL, sleep quality $(r=0.38)$, perceived fatigue ( $r=0.28)$, depression ( $r=0.38)$ and anxiety $(r=0.28)$. The relationships between RLS severity and the domains of HRQOL were attenuated when accounting for fatigue, depression and/or anxiety. Worse RLS severity was associated with reduced HRQOL, which was accounted for by fatigue, depression and anxiety.
\end{abstract}

\section{KEYWORDS}

anxiety, depression, fatigue, health-related quality of life, multiple sclerosis, restless legs

syndrome

\section{1 | INTRODUCTION}

Restless legs syndrome (RLS) is a sleep disorder characterized by uncomfortable sensations and an intense, often uncontrollable, urge to move the legs (Restless Legs Syndrome Foundation, 2018). The defining features of RLS include: (a) an urge to move the extremities that is accompanied by uncomfortable or unpleasant sensations; (b) the sensations are partially or totally relieved by movement; (c) the sensations begin or worsen during periods of rest or inactivity; and (d) the sensations worsen in the evening (Walters et al., 2003). RLS occurs in upwards of $15 \%$ of the general population (Ohayon, O'hara,
\& Vitiello, 2012), and the prevalence is substantially higher among persons with multiple sclerosis (MS), with estimates as high as $65 \%$ (Sieminski, Losy, \& Partinen, 2015). This presents RLS as one of the most common, and potentially burdensome, sleep disorders among persons with MS (Braley \& Chervin, 2015).

Restless legs syndrome may exacerbate many of the symptoms and consequences of MS and could have implications for health-related quality of life (HRQOL). Persons with MS who have RLS report greater daytime sleepiness, lower sleep quality, worse clinical disability (Manconi et al., 2008; Moreira et al., 2008) and greater symptoms of depression and fatigue (Aydar, Kurt, Karaer Unaldi, \& 
Erkorkmaz, 2011; Moreira et al., 2008) than those with MS who do not have RLS. Such co-occurring consequences of RLS are common influences of HRQOL in MS (Benito-Leon, Morales, Rivera-Navarro, $\&$ Mitchell, 2003), yet there is limited evidence regarding the nature of the relationship between RLS and HRQOL in MS.

The present study examined the relationship between RLS and HRQOL in people with MS. We compared common symptoms and consequences associated with MS between groups with and without RLS (i.e., MS + RLS and MS groups, respectively) and further examined the association between RLS severity, MS-related outcomes and HRQOL in people with both MS and RLS. This was followed by a search for possible intermediary factors in the relationship, including disability status, sleep quality, fatigue, depression and anxiety in adults with both MS and RLS. We hypothesized that those with MS and RLS (i.e., the MS + RLS group) would report worse HRQOL, clinical disability, sleep quality, fatigue, depression and anxiety than those with MS alone (i.e., the MS group). We further hypothesized that the relationship between RLS severity and HRQOL in those with MS and RLS might be accounted for by the aforementioned co-occurring consequences of RLS (i.e., sleep quality, clinical disability status, fatigue and depression).

\section{2 | MATERIALS AND METHODS}

\section{1 | Participants}

We recruited a sample of persons with MS through the North American Research Committee on Multiple Sclerosis (NARCOMS) patient registry. The random sample of 1,000 persons with MS received printed letters from NARCOMS staff, and the sample size was selected based on an expectation of $25 \%$ participation in the actual study, which was estimated by NARCOMS. Those who were interested in participating contacted the research team through Email or telephone, and members of the research team conducted a brief screening interview for inclusion. The inclusion criteria were: (a) age 18 years or older; (b) self-reported diagnosis of MS; (c) member of the NARCOMS registry; and (d) willingness to complete the questionnaires and return the materials via the United States Postal Service (USPS). Of the 1,000 persons with MS recruited through NARCOMS, 316 made contact with the research team and 296 were screened for eligibility (Figure 1); one person declined to participate after the description of the study. The research team distributed study materials to 295 persons and
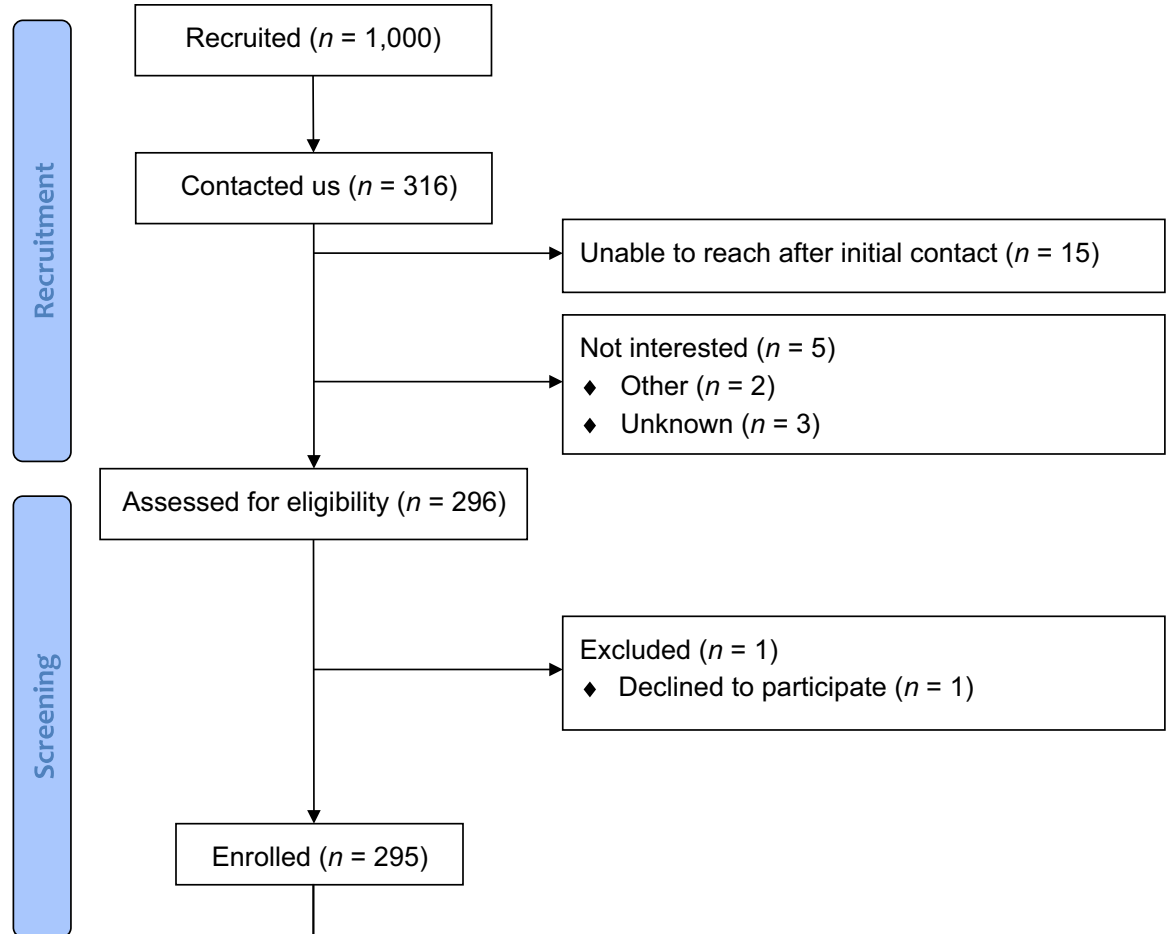

Assessed for eligibility $(n=296)$

Excluded $(n=1)$

- Declined to participate $(n=1)$

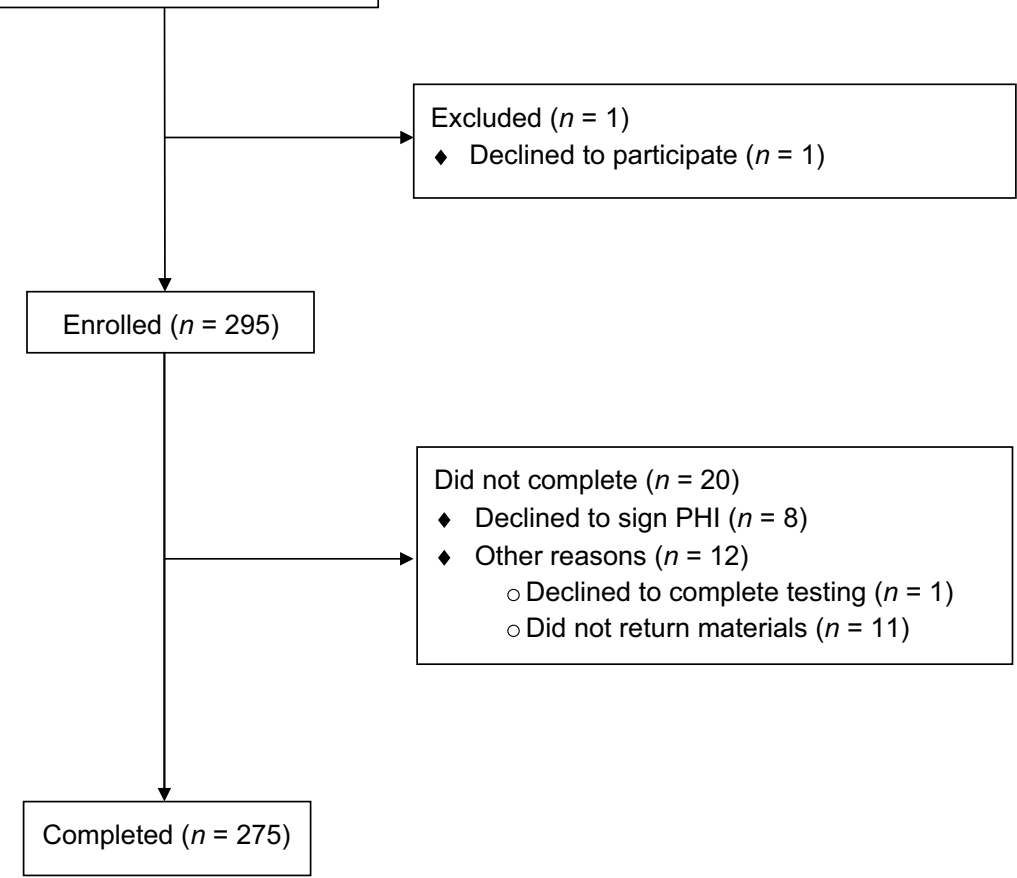

FIGURE 1 CONSORT diagram. PHI, Protected Health Information form 
284 individuals returned study materials to the research team. Of those who returned materials, nine declined to participate and 11 persons did not return packets for unknown reasons. The final sample consisted of 275 persons with MS.

\section{2 | Restless legs syndrome}

\subsection{1 | Diagnosis}

The diagnosis of RLS was based on the Cambridge-Hopkins Restless Legs Syndrome Questionnaire (CH-RLSq), which has demonstrated validity and sensitivity for diagnosing RLS via a survey (Allen, Burchell, Macdonald, Hening, \& Earley, 2009). The CH-RLSq requires that participants fulfill the five criteria for a positive diagnosis of RLS and further includes items to help exclude common mimics of RLS (i.e., leg cramps and positional discomfort). These items consisted of five yes or no questions, two questions assessing when symptoms occur (i.e., at rest or when active, as well as the time of day) and one question for assessing if a single occurrence of movement relieves the symptoms (i.e., usually relieves, does not usually relieve, or don't know). Items were reviewed by a researcher and scored as positive for RLS (i.e., the MS + RLS group) if responses were consistent with the aforementioned RLS diagnostic criteria or as negative for RLS (i.e. the MS group) if any item was inconsistent with RLS diagnostic criteria.

\subsection{2 | Symptom severity}

The severity of symptoms of the restless legs syndrome was measured using the International Restless Legs Syndrome Study Group (IRLS) Scale. The IRLS is a validated 10 -question survey that provides a global score commonly used to assess the overall severity of symptoms over the previous 7 days (Walters et al., 2003). Items were rated on a scale ranging from 0 to 4 and individual item scores were summed for a measure of overall symptom severity ranging from 0 to 40 . IRLS scores were further categorized into no symptoms (score of 0), mild (score 1-10), moderate (score 11-20), severe (score 21-30) and very severe (score 31-40) (Walters et al., 2003). The IRLS has demonstrated validity, reliability and responsiveness in clinical trial settings (Abetz et al., 2006).

\section{3 | Health-related quality of life}

The Multiple Sclerosis Impact Scale (MSIS-29) provides a diseasespecific measure of HRQOL (Hobart, Lamping, Fitzpatrick, Riazi, \& Thompson, 2001). The MSIS-29 measures physical and psychological aspects of HRQOL using 29 separate items rated on a five-point Likert scale from 1 (not at all) to 5 (extremely) with regard to the past 4 weeks. Respective items were utilized to generate subscale scores for the physical and psychological domains of HRQOL. The physical domain was scored by summing the scores for items $1-20$, subtracting 20 , dividing the difference by 80 , and then multiplying the result by 100 . The psychological domain was scored by summing the scores for items 21 to 29 , subtracting nine, dividing the difference by 36 and then multiplying by 100 . Higher scores on both subscales represent a greater impact of MS on HRQOL during the previous 2 weeks. Reliability and validity of the MSIS-29 have been demonstrated in subjects with MS (Learmonth, Hubbard, Mcauley, \& Motl, 2014).

\section{4 | Demographics and clinical characteristics}

Participants completed a demographic and clinical characteristics questionnaire for information regarding age, sex, race, MS subtype and disease duration. Participants further completed the Patient Determined Disease Steps (PDDS), a single item measure of self-reported disability status (Hohol, Orav, \& Weiner, 1995, 1999). PDDS scores ranged between 0 (normal) and 8 (bedridden), and the scores have demonstrated evidence for validity and strong correlations with the Expanded Disability Status Scale (EDSS), pyramidal and cerebellar functional scores and walking ability in persons with MS (Learmonth et al., 2013).

\section{5 | Sleep quality}

Subjective sleep quality was assessed with the Pittsburgh Sleep Quality Index (PSQI) (Buysse, Reynolds, Monk, Berman, \& Kupfer, 1989). The PSQI consists of 19 items that measure the quality of one's sleep and sleep disturbances over the past month. The items include a number of factors associated with sleep quality, including subjective sleep quality, duration of sleep, sleep latency, habitual sleep efficiency (i.e., frequency and severity of sleep-related problems, including breathing difficulty, pain, urinary frequency), use of sleeping medications, and the impact of poor sleep on daily functioning. Components were scored from 0 (fairly good) to 3 (very bad) and component scores are summed for a global score ranging from 0 to 21 ; higher scores reflect worse sleep quality. Of note, scores greater than 5 are indicative of severe difficulties in at least two domains or moderate difficulties in more than three areas and the PSQI has demonstrated evidence for validity and reliability in assessing sleep quality in the general population (Buysse et al., 1989).

\subsection{Fatigue symptoms}

Perceived fatigue was measured using the Fatigue Severity Scale (FSS) (Krupp, Larocca, Muir-Nash, \& Steinberg, 1989). The FSS contains nine Likert-scale items that assess fatigue symptoms during the past week, rated on a 7-point scale ranging between 1 (strongly disagree) and 7 (strongly agree). Items were averaged for an overall measure of a person's severity of fatigue that ranges between 1 and 7. Of note, FSS scores of 4 (or total scores of 36) or above indicate severe MS-related fatigue (Andreasen, Stenager, \& Dalgas, 2011). There is evidence for the internal consistency, test-retest reliability, validity and precision of FSS scores as a measure of fatigue (Krupp et al., 1989).

\subsection{Anxiety and depression symptoms}

The Hospital Anxiety and Depression Scale (HADS) contains 14 items that measure perceived symptoms of depression and anxiety over the 
past 4 weeks (Zigmond \& Snaith, 1983). Items were individually rated on a 4-point scale ranging from 0 (most of the time) to 3 (not at all). A subset of seven items were reverse-scored per subscale and summed into a subscale score for symptoms of depression (HADS-D) or symptoms of anxiety (HADS-A) that ranged from 0 to 21; higher scores reflected a greater frequency of depressive and anxiety symptoms. Of note, a cut-point score of 7 has been identified for depression and anxiety screening and a score of 11 suggests major depression and generalized anxiety disorder in individuals with MS (Marrie et al., 2018). Additionally, the HADS has demonstrated evidence for the reliability and validity of these scores in people with MS (Marrie et al., 2018).

\subsection{Procedures}

This study was approved by the university's Institutional Review Board and all participants provided written informed consent. After initial telephone contact and screening, all participants who verbally volunteered were mailed a packet containing the informed-consent document, questionnaire battery, and a prestamped and preaddressed envelope for return service through the USPS. The participants were instructed to complete the battery of questionnaires over a 7-day period. After completing the questionnaires, participants returned a signed copy of the informed consent along with the study materials through the USPS. All participants received $\$ 10$ for voluntary participation.

\section{9 | Statistical analysis}

All statistical analyses were conducted using SPSS version 24 and descriptive data are presented as mean scores along with the standard deviations (SDs), unless otherwise specified. We examined skewness and kurtosis estimates and frequency distributions for establishing normality of the variables; the skewness and kurtosis estimates along with inspection of frequency distributions did not identify problems with normality for any of the main study variables (i.e., age, sex, race, MS type, disease duration, PDDS, IRLS, MSIS, PSQI, FSS and HADS). The differences in outcome measures between groups (MS + RLS vs. MS) were therefore determined using an independent samples $t$ test for continuous variables, the MannWhitney U-test for categorical variables and the chi-squared test for nominal variables. Cohen's $d$ was utilized to estimate the magnitude of difference between groups and effect sizes of $0.2,0.5$ and 0.8 were interpreted as small, moderate and large, respectively (Cohen, 1988). Within the MS + RLS group, the associations among RLS severity, physical and mental components of HRQOL and MSrelated consequences (i.e., disability status, sleep quality, fatigue, depression and anxiety) were examined by means of Pearson product $(r)$ correlation coefficients; values of $0.1,0.3$ and 0.5 were interpreted as small, moderate and large, respectively (Cohen, 1988).

We evaluated the relationship between RLS diagnosis and physical and psychological HRQOL scores using multivariate linear regressions with forward stepwise selection $(\alpha=0.05)$. We regressed $\mathrm{CH}$-RLSq on each domain of HRQOL (i.e., physical and psychological) in Step 1, and CH-RLSq plus variables that were significantly different between groups (i.e. MS + RLS vs. MS) with the HRQOL domains in Step 2. We examined the change in the standardized beta-coefficient for CH-RLSq and the domain of HRQOL between Steps 1 and 2 for judging the variables that may be intermediary factors in the associations within the total sample of participants. Within the MS + RLS group, we examined potential intermediary variables in the relationship between RLS severity and physical and psychological HRQOL scores using multivariate linear regressions with forward stepwise selection $(\alpha=0.05)$. We regressed the total IRLS score on each domain of HRQOL in Step 1, and RLS severity plus variables that were significantly associated with the HRQOL domain in Step 2. We examined the change in the standardized beta-coefficient for IRLS and the domain of HRQOL between Steps 1 and 2 for judging the variables that may be intermediary factors in the associations within those with MS + RLS. A p-value of less than 0.05 was adopted for determining statistical significance with all inferential analyses.

\section{RESULTS}

\section{1 | Overall sample characteristics}

Demographic and clinical characteristics for the overall sample of participants $(n=275)$ are presented in Table 1 . The sample was largely female (81\%) and Caucasian (94.9\%), with a mean age of $60 \pm 10$ years. Most participants had RRMS (65.6\%) with moderate disability (median [interquartile range] PDDS score: 3.0 [5.0]), an average disease duration of $20 \pm 9.7$ years, and most participants were using an MS-specific disease-modifying therapy (DMT; 84.3\%). The sample reported scores consistent with moderate-to-severe sleep disturbances (PSQI global score, 7.2 \pm 3.6 ) and severe MS-related fatigue (FSS: $4.7 \pm 1.8$ ), based on cut-off scores of greater than 5 (Buysse et al., 1989) and greater than 4 (Andreasen et al., 2011) respectively.

\subsection{1 | Restless legs syndrome in multiple sclerosis}

Demographic and clinical characteristics of subsamples of MS + RLS $(n=74)$ and MS only $(n=201)$ are presented in Table 1. Approximately $27 \%$ of our sample fit the criteria for a positive diagnosis of RLS based on the CH-RLSq diagnostic questionnaire. Within the MS + RLS group, nine (12.2\%) individuals reported no symptoms, 26 (35.1\%) mild symptoms, 28 (37.8\%) moderate symptoms, 10 (13.5\%) severe symptoms, and one (1.4\%) reported very severe symptoms of RLS. Of note, 20 (27\%) participants with MS + RLS were taking medications that can reduce symptoms of RLS and 14 (18.9\%) were taking medications that can exacerbate symptoms of RLS (Buchfuhrer, 2012; Restless Legs Syndrome Foundation, 2018). The two groups (i.e., MS + RLS and MS) were not significantly different in age, sex, race, MS type, disease duration, self-reported clinical disability status, number of participants on DMTs, sleep quality or depression symptoms.

The MS + RLS group reported significantly worse HRQOL in both domains (MSIS-physical, $p=0.020$; MSIS-psychological, $p=0.007$ ). The 
TAB LE 1 Participant characteristics for all participants $(N=275)$ and the subsamples of participants with and without restless legs syndrome

\begin{tabular}{|c|c|c|c|c|c|}
\hline & $\begin{array}{l}\text { All participants } \\
(N=275)\end{array}$ & MS + RLS $(n=74)$ & $\mathrm{MS}(n=201)$ & $p$-value & $d$ \\
\hline Age, years & $59.7 \pm 10.1$ & $59.4 \pm 9.9$ & $59.9 \pm 10.2$ & 0.748 & - \\
\hline Race, $n(\%)$ & & & & $0.422^{\mathrm{a}}$ & - \\
\hline Caucasian & 261 (94.9\%) & 73 (98.6\%) & $188(93.4 \%)$ & - & - \\
\hline Latino/Latina & $5(1.8 \%)$ & $0(0.0 \%)$ & $5(0.3 \%)$ & - & - \\
\hline Other & $3(1.1 \%)$ & $1(1.4 \%)$ & 2 (1.0\%) & - & - \\
\hline MS type, $n(\%)$ & & & & $0.731^{\mathrm{a}}$ & - \\
\hline Benign & $3(1.1 \%)$ & $0(0.0 \%)$ & $3(1.5 \%)$ & - & - \\
\hline Disease duration, years & $20.4 \pm 9.7$ & $20.0 \pm 9.7$ & $20.5 \pm 9.7$ & 0.686 & - \\
\hline MS DMT, $n(\%)$ & 231 (84.3\%) & $53(71.6 \%)$ & 149 (74.1\%) & $0.676^{a}$ & - \\
\hline PDDS, median (IQR) & $3.0(5)$ & $3.5(3)$ & $3.0(5)$ & $0.291^{b}$ & - \\
\hline IRLS & $7.6 \pm 8.0$ & $11.4 \pm 7.6$ & $6.2 \pm 7.7$ & 0.000 & 0.680 \\
\hline RLS modifying Rx & $69(25.1 \%)$ & $20(27.0 \%)$ & 49 (24.4\%) & $0.653^{\mathrm{a}}$ & - \\
\hline RLS exacerbating Rx & 39 (14.2\%) & 14 (18.9\%) & $25(12.4 \%)$ & $0.172^{\mathrm{a}}$ & - \\
\hline MSIS-physical & $35.0 \pm 25.0$ & $40.7 \pm 24.6$ & $32.8 \pm 24.8$ & 0.020 & 0.319 \\
\hline MSIS-psychological & $25.3 \pm 19.1$ & $30.4 \pm 22.7$ & $23.4 \pm 17.3$ & 0.007 & 0.347 \\
\hline
\end{tabular}

Data are presented as mean \pm standard deviation unless otherwise specified. Bolded values are indicative of significant differences between groups. Abbreviations: MS, multiple sclerosis; RLS, restless legs syndrome; RRMS, relapsing-remitting MS; SPMS, secondary progressive MS; PPMS, primary progressive MS; PDDS, Patient Determined Disease Status; IQR, interquartile range; IRLS, International Restless Legs Syndrome Study Group Scale; MSIS, Multiple Sclerosis Impact Scale; PSQI, Pittsburgh Sleep Quality Index; FSS, Fatigue Severity Scale; HADS-D, Hospital Anxiety and Depression Scale-Depression domain; HADS-A, Hospital Anxiety and Depression Scale-Anxiety domain.

${ }^{\text {a } C h i-s q u a r e ~ a n a l y s i s . ~}$

${ }^{b}$ Wilcoxon-Mann-Whitney test.

MS + RLS group reported significantly greater perceived fatigue (FSS, $p=0.007$ ) and anxiety symptoms (HADS-A, $p=0.042$ ) than the MS-only group. Additionally, the MS + RLS group had a positive screening for anxiety and depression based on a cut-off score of 7 (Marrie et al., 2018). The difference between groups was small for the physical $(d=0.32)$ and psychological $(d=0.35)$ domains of HRQOL, perceived fatigue $(d=0.40)$, depression symptoms $(d=0.21)$ and anxiety symptoms $(d=0.25)$.

\section{2 | Correlation analysis}

The bivariate correlations among RLS severity, HRQOL, clinical disability status, sleep quality, fatigue, depression and anxiety in the MS + RLS group are presented in Table 2. RLS severity was significantly associated with physical $(r=0.43)$ and psychological ( $r=0.46)$ HRQOL, sleep quality ( $r=0.38)$, perceived fatigue $(r=0.28)$ and symptoms of depression $(r=0.38)$ and anxiety $(r=0.28)$. The physical domain of HRQOL was significantly associated with clinical disability status $(r=0.74)$, sleep quality $(r=0.33)$, fatigue $(r=0.68)$ and depression ( $r=0.60$ ); the psychological domain of HRQOL was significantly associated with sleep quality $(r=0.56)$, fatigue $(r=0.47)$, depression ( $r=0.78)$ and anxiety $(r=0.74)$.

\section{3 | Linear regression analysis}

The summary of linear regression analyses for evaluating the relationship between RLS diagnosis and HRQOL in the total sample 
TAB LE 2 Summary of Pearson product bivariate correlations in adults with restless legs syndrome and multiple sclerosis $(n=74)$

\begin{tabular}{|c|c|c|c|c|c|c|c|}
\hline & IRLS & MSIS-Physical & MSIS-Psychological & PDDS & PSQI & FSS & HADS-D \\
\hline MSIS-physical & $0.429^{b}$ & - & & & & & \\
\hline MSIS- psychological & $0.463^{b}$ & $0.563^{b}$ & - & & & & \\
\hline PDDS & 0.194 & $0.737^{b}$ & 0.075 & - & & & \\
\hline PSQI & $0.382^{b}$ & $0.334^{b}$ & $0.561^{b}$ & 0.096 & - & & \\
\hline FSS & $0.277^{a}$ & $0.678^{b}$ & $0.474^{b}$ & $0.363^{b}$ & $0.409^{b}$ & - & \\
\hline HADS-D & $0.375^{b}$ & $0.599^{b}$ & $0.776^{b}$ & $0.284^{a}$ & $0.443^{b}$ & $0.437^{b}$ & - \\
\hline HADS-A & $0.278^{a}$ & 0.202 & $0.740^{b}$ & -0.168 & $0.383^{b}$ & 0.102 & $0.562^{b}$ \\
\hline
\end{tabular}

Abbreviations: IRLS, International Restless Legs Syndrome Study Group Scale; MSIS, Multiple Sclerosis Impact Scale; PDDS, Patient Determined Disease Status; PSQI, Pittsburgh Sleep Quality Index; FSS, Fatigue Severity Scale; HADS-D, Hospital Anxiety and Depression Scale-Depression domain; HADS-A, Hospital Anxiety and Depression Scale-Anxiety domain.

${ }^{\mathrm{a}} p<0.05$.

${ }^{\mathrm{b}} p<0.01$.

( $n=275$ ) is presented in Table 3. RLS diagnosis significantly predicted the physical domain of HRQOL in Step 1 ( $F=5.310, p=0.022$; $\left.R^{2}=0.019\right)$, and this relationship was accounted for by FSS and HADS-A in Step 2 ( $F=29.564, p=0.000$; $R$ change $=0.228$ ). RLS diagnosis significantly predicted the psychological domain of HRQOL in Step $1\left(F=7.362, p=0.007 ; R^{2}=0.026\right)$, and this relationship was accounted for by FSS and HADS-A in Step 2 ( $F=109.293, p=0.000$; $R^{2}$ change $=0.522$.

TA B LE 3 Summary of linear regression analysis for restless legs syndrome diagnosis and other variables predicting the physical and psychological domains of health-related quality of life in adults with multiple sclerosis $(n=275)$

\begin{tabular}{|c|c|c|c|}
\hline & B & SE B & $\boldsymbol{\beta}$ \\
\hline \multicolumn{4}{|l|}{ MSIS-physical } \\
\hline \multicolumn{4}{|l|}{ Step 1} \\
\hline $\mathrm{CH}-\mathrm{RLSq}$ & 7.764 & 3.369 & $0.138^{a}$ \\
\hline \multicolumn{4}{|l|}{ Step 2} \\
\hline $\mathrm{CH}-\mathrm{RLSq}$ & 3.036 & 3.019 & 0.054 \\
\hline FSS & 6.684 & 0.756 & $0.476^{\mathrm{a}}$ \\
\hline HADS-A & 0.482 & 0.494 & 0.052 \\
\hline \multicolumn{4}{|c|}{$R^{2}=0.019$ for step $1 ; \Delta R^{2}=0.228$ for step $2 .{ }^{a} p<0.05$} \\
\hline \multicolumn{4}{|c|}{ MSIS-psychological } \\
\hline \multicolumn{4}{|l|}{ Step 1} \\
\hline $\mathrm{CH}-\mathrm{RLSq}$ & 6.975 & 2.571 & $0.162^{\mathrm{a}}$ \\
\hline \multicolumn{4}{|l|}{ Step 2} \\
\hline $\mathrm{CH}-\mathrm{RLSq}$ & 1.393 & 1.791 & 0.032 \\
\hline FSS & 3.695 & 0.449 & $0.343^{a}$ \\
\hline HADS-A & 4.297 & 0.293 & $0.607^{\mathrm{a}}$ \\
\hline \multicolumn{4}{|c|}{$R^{2}=0.026$ for step $1 ; \Delta R^{2}=0.522$ for step $2 .{ }^{a} p<0.05$} \\
\hline
\end{tabular}

Abbreviations: MSIS, Multiple Sclerosis Impact Scale; CH-RLSq, Cambridge-Hopkins Restless Legs Syndrome Questionnaire; FSS, Fatigue Severity Scale; HADS-A, Hospital Anxiety and Depression Scale-Anxiety domain.

${ }^{a} p<0.05$.
The summary of linear regression analyses for evaluating variables that may be intermediary in the relationship between RLS severity and HRQOL in those with MS + RLS $(n=74)$ is presented in Table 4. RLS severity significantly predicted the physical domain of HRQOL in Step $1\left(F=16.259, p=0.000 ; R^{2}=0.184\right)$, and this relationship was partially accounted for by FSS and HADS-D in Step 2 $\left(F=26.626, p=0.000 ; R^{2}\right.$ change $\left.=0.423\right)$. RLS severity significantly predicted the psychological domain of HRQOL in Step $1(F=19.646$, $\left.p=0.000 ; R^{2}=0.214\right)$, and this relationship was partially accounted for by FSS, HADS-D and HADS-A in Step 2 ( $F=37.113, p=0.000$; $R^{2}$ change $\left.=0.596\right)$.

\section{4 | DISCUSSION}

This study provided a novel evaluation of the relationship between RLS severity and HRQOL, and identified potential intermediary variables in this relationship for persons with MS and RLS. Persons with MS and RLS had reduced HRQOL in both the physical and psychological domains. Consistent with previous research, persons with MS and RLS experienced more severe fatigue and a greater frequency of anxiety symptoms than those without RLS (Cederberg \& Motl, 2016; Giannaki et al., 2018). Among those with MS and RLS, RLS severity was moderately correlated with HRQOL, whereby greater RLS severity was associated with a greater impact of MS on both physical and psychological domains of HRQOL. The relationships between RLS severity and the domains of HRQOL were attenuated when accounting for fatigue, depression and/or anxiety, suggesting that these symptoms may be intermediary factors in the association between RLS severity and HRQOL.

Within this study of adults with MS, meeting the diagnostic criteria for RLS was associated with significantly worse physical and psychological HRQOL, fatigue, anxiety and depression, thereby suggesting that the presence of RLS in adults with MS may negatively impact HRQOL and associated outcomes. The presence of RLS significantly predicted the physical and psychological domains 
TAB LE 4 Summary of linear regression analysis for restless legs syndrome severity and other variables predicting the physical and psychological domains of health-related quality of life in adults with restless legs syndrome and multiple sclerosis $(n=74)$

\begin{tabular}{|c|c|c|c|}
\hline & B & SE B & $\boldsymbol{\beta}$ \\
\hline \multicolumn{4}{|l|}{ MSIS-physical } \\
\hline \multicolumn{4}{|l|}{ Step 1} \\
\hline IRLS & 1.393 & 0.345 & $0.429^{a}$ \\
\hline \multicolumn{4}{|l|}{ Step 2} \\
\hline IRLS & 0.641 & 0.275 & $0.198^{a}$ \\
\hline PSQI & -0.647 & 0.548 & -0.105 \\
\hline FSS & 7.508 & 1.271 & $0.515^{\mathrm{a}}$ \\
\hline HADS-D & 2.686 & 0.704 & $0.343^{\mathrm{a}}$ \\
\hline \multicolumn{4}{|c|}{$R^{2}=0.184$ for step $1 ; \Delta R^{2}=0.423$ for step $2 .{ }^{a} p<0.05$} \\
\hline \multicolumn{4}{|c|}{ MSIS-psychological } \\
\hline \multicolumn{4}{|l|}{ Step 1} \\
\hline IRLS & 1.383 & 0.312 & $0.463^{\mathrm{a}}$ \\
\hline \multicolumn{4}{|l|}{ Step 2} \\
\hline IRLS & 0.338 & 0.178 & 0.113 \\
\hline PSQI & 0.512 & 0.363 & 0.090 \\
\hline FSS & 2.809 & 0.852 & $0.209^{a}$ \\
\hline HADS-D & 2.488 & 0.532 & $0.345^{\mathrm{a}}$ \\
\hline HADS-A & 3.410 & 0.499 & $0.461^{a}$ \\
\hline \multicolumn{4}{|c|}{$R^{2}=0.214$ for step $1 ; \Delta R^{2}=0.594$ for step $2 .{ }^{a} p<0.05$} \\
\hline
\end{tabular}

Abbreviations: MSIS, Multiple Sclerosis Impact Scale; IRLS, International Restless Legs Syndrome Study Group Scale; PSQI, Pittsburgh Sleep Quality Index; FSS, Fatigue Severity Scale; HADS-D, Hospital Anxiety and Depression Scale-Depression domain; HADS-A, Hospital Anxiety and Depression Scale-Anxiety domain.

${ }^{a} p<0.05$.

of HRQOL, yet our results suggest that the severity of fatigue and frequency of anxiety symptoms partially account for such relationships. This further suggests that fatigue and symptoms of anxiety may be important intermediaries in the relationship between RLS diagnosis and HRQOL in adults with MS.

Our results indicated that anxiety was significantly worse in adults with MS and RLS and that anxiety was positively associated with RLS severity and the psychological domain of HRQOL. To date, this is the first study to evaluate symptoms of anxiety in adults with MS and RLS. Anxiety is characterized by excessive worry that an individual finds difficult to control, which can cause significant problems in other areas of life (National Institute of Mental Health, 2018). Results from the current study suggest that anxiety may be an important factor in the relationships among RLS, RLS severity and HRQOL; however, the pathological connection between RLS and anxiety is unclear. One potential explanation is that RLS shares similar characteristics with anxiety disorders, wherein the diagnostic criteria for generalized anxiety disorder include symptoms of restlessness, fatigue, difficulty concentrating, irritability, muscle tension and sleep disturbance (Patriquin \& Mathew, 2017). Thus, the unpredictable and uncontrollable nature of RLS symptoms may contribute to or exacerbate anxiety symptoms, which in turn impact HRQOL in adults with MS. More research is necessary to further evaluate the temporal relationship between RLS severity and anxiety, as well as the impact of anxiety in persons with MS and RLS.

Results from the current study indicated that a reduced HRQOL in the physical domain was associated with worse RLS severity, greater self-reported clinical disability, poorer sleep quality, greater fatigue severity and a higher frequency of depression. Similarly, reduced HRQOL in the psychological domain was associated with worse RLS severity, poorer sleep quality, greater fatigue and a greater frequency of depression and anxiety symptoms. These results are similar to previous literature that has demonstrated significant correlations between HRQOL and fatigue, poor sleep (Lobentanz et al., 2004; Amato et al., 2001, Boe Lunde et al., 2012; Tabrizi \& Radfar, 2015; Kotterba et al., 2018), depression (Amato et al., 2001; Lobentanz et al., 2004) and anxiety (Fruehwald, Loeffler-Stastka, Eher, Saletu, \& Baumhackl, 2001) in persons with MS. Importantly, this is the first study to identify severity of RLS as an important correlate of HRQOL and this association may be accounted for by fatigue, depression and anxiety. These results build upon current literature that identifies depression and fatigue as strong predictors of HRQOL in persons with MS (Amato et al., 2001; Fruehwald et al., 2001; Lobentanz et al., 2004; Nourbakhsh, Julian, \& Waubant, 2016). Future research should consider other possible factors that may mediate the relationship between the severity of RLS symptoms and HRQOL in persons with MS.

\section{5 | LIMITATIONS}

There are important limitations to consider when interpreting our results. The cross-sectional design of this study precludes any inferences of causality or temporality. The response rate from the NARCOMS registry was low (i.e., 31\%) and further reduced (27\%) based on valid questionnaires. Thus, selection bias may have played a role in our results. As this was a mail-based protocol, all measures are self-report in nature. The lack of an interview for RLS diagnosis may have led to false negative or false positive diagnoses on the $\mathrm{CH}$-RLSq. However, the RLS diagnostic questionnaire (i.e., $\mathrm{CH}-\mathrm{RLSq}$ ) has been validated for diagnosing RLS, with consideration of certain RLS mimics, in the general population (Allen et al., 2009). We did not include criteria for a minimum time since diagnosis. Some prescription medications for MS present another limitation to assessing RLS symptom severity in this population, as a number of prescriptions for symptoms and consequences of MS can be used to treat symptoms of RLS (e.g., pramipexole, gabapentin and rotigotine; Restless Legs Syndrome Foundation, 2018) or may exacerbate symptoms of RLS (e.g., antidepressants, first-generation antihistamines; Buchfuhrer, 2012); 25.1\% ( $n=69)$ of our sample with MS and RLS were taking a medication that could reduce RLS severity and $14.2 \%(n=39)$ were taking medications that could exacerbate RLS severity. This paper included only a single 
measure for the evaluation of sleep, but it did not include a measure for assessing daytime sleepiness (e.g., the Epworth Sleepiness Scale). This is a limitation as daytime sleepiness is a common correlate of RLS that may impact HRQOL. Our sample consisted primarily of persons with RRMS (65.8\%), suggesting that our sample may not be fully representative of the MS population, and we further note that some subjects may be unaware of a transition from RRMS to secondary progressive MS (SPMS). Additionally, this high prevalence of individuals with RRMS may not be representative of the MS + RLS population as prior literature suggests that RLS may be more prevalent in those with PPMS and those with greater levels of disability (Manconi et al., 2007, 2008). However, we included a large, random sample of individuals with MS that included al phenotypes of MS and our sample was representative of US peak prevalence (i.e., 55-64 years) for age and sex (Wallin et al., 2019), disease duration and MS type (Multiple Sclerosis International Foundation, 2013).

\section{6 | CONCLUSIONS}

This study sheds new light on the relationships among RLS severity, MS symptomology and physical and psychological domains of HRQOL. Persons with MS and RLS experience reduced HRQOL in both physical and psychological domains, worse fatigue and more frequent symptoms of depression and anxiety. Greater severity of RLS was associated with both physical and psychological domains of HRQOL, which was seemingly accounted for by fatigue, depression and anxiety. These results identify a possible pathway for mitigating reductions in $\mathrm{HRQOL}$ through the reduction of RLS and other MS symptoms (e.g., fatigue and anxiety). The identification of intermediary factors in the relationship between RLS and QOL in MS provides valuable information that could be utilized to improve symptomatic treatment and modulate treatment over time, consequently improving HRQOL, in persons with MS and RLS. Future research should further examine the role of anxiety in persons with MS and RLS, as well as identify correlates and factors that may mediate the relationship between RLS and HRQOL, as early identification and treatment of modifiable factors, such as fatigue, depression and anxiety, may improve quality of life in people with MS and RLS.

\section{ACKNOWLEDGEMENTS}

None.

\section{CONFLICT OF INTEREST}

TJB reports funding outside the submitted work. TJB is also named in a provisional patent held by the University of Michigan, concerning treatment for sleep apnea, and serves as a consultant/advisor for Jazz Pharmaceuticals. ASW reports grants outside the submitted work. RWM reports grants from NMSS, during the conduct of the study. KLJC, BJ and JES have nothing to disclose.

\section{AUTHOR CONTRIBUTION}

RWM was responsible for study conception and design. KLJC, BJ and JES were responsible for data acquisition. KJLC was responsible for data analysis and interpretation and drafting the manuscript with the guidance of RWM. TJB and ASW, along with all other co-authors, provided critical revisions.

\section{ORCID}

Katie L. J. Cederberg (iD https://orcid.org/0000-0001-5693-9293

\section{REFERENCES}

Abetz, L., Arbuckle, R., Allen, R. P., Garcia-Borreguero, D., Hening, W., Walters, A. S., ... Kirsch, J. M. (2006). The reliability, validity and responsiveness of the International Restless Legs Syndrome Study Group rating scale and subscales in a clinical-trial setting. Sleep Medicine, 7, 340-349. https://doi.org/10.1016/j.sleep.2005.12.011

Allen, R. P., Burchell, B. J., Macdonald, B., Hening, W. A., \& Earley, C. J. (2009). Validation of the self-completed Cambridge-Hopkins questionnaire (CH-RLSq) for ascertainment of restless legs syndrome (RLS) in a population survey. Sleep Medicine, 10, 1097-1100. https:// doi.org/10.1016/j.sleep.2008.10.007

Amato, M. P., Ponziani, G., Rossi, F., Liedl, C. L., Stefanile, C., \& Rossi, L. (2001). Quality of life in multiple sclerosis: The impact of depression, fatigue and disability. Multiple Sclerosis Journal, 7, 340-344. https:// doi.org/10.1177/135245850100700511

Andreasen, A. K., Stenager, E., \& Dalgas, U. (2011). The effect of exercise therapy on fatigue in multiple sclerosis. Multiple Sclerosis Journal, 17, 1041-1054. https://doi.org/10.1177/1352458511401120

Aydar, G., Kurt, S., Karaer Unaldi, H., \& Erkorkmaz, U. (2011). Restless legs syndrome in multiple sclerosis. European Neurology, 65, 302306. https://doi.org/10.1159/000327315

Benito-Leon, J., Morales, J. M., Rivera-Navarro, J., \& Mitchell, A. (2003). A review about the impact of multiple sclerosis on health-related quality of life. Disability and Rehabilitation, 25, 1291-1303. https:// doi.org/10.1080/09638280310001608591

Braley, T. J., \& Chervin, R. D. (2015). A practical approach to the diagnosis and management of sleep disorders in patients with multiple sclerosis. Therapeutic Advances in Neurological Disorders, 8, 294-310. https ://doi.org/10.1177/1756285615605698

Buchfuhrer, M. J. (2012). Strategies for the treatment of restless legs syndrome. Neurotherapeutics: the Journal of the American Society for Experimental NeuroTherapeutics, 9,776-790. https://doi.org/10.1007/ s13311-012-0139-4

Buysse, D. J., Reynolds, C. F. 3rd, Monk, T. H., Berman, S. R., \& Kupfer, D. J. (1989). The Pittsburgh Sleep Quality Index: A new instrument for psychiatric practice and research. Psychiatry Research, 28, 193-213. https://doi.org/10.1016/0165-1781(89)90047-4

Cederberg, K. L., \& Motl, R. W. (2016). Restless legs syndrome in multiple sclerosis: A call for better understanding and non-pharmacological management. Current Trends in Neurology, 10, 65-73.

Cohen, J. (1988). Statistical power analysis for the behavioral sciences. Lawrence Earlbaum Associates (pp. 20-26). Hillsdale: NJ.

Fruehwald, S., Loeffler-Stastka, H., Eher, R., Saletu, B., \& Baumhackl, U. (2001). Depression and quality of life in multiple sclerosis. Acta Neurologica Scandinavica, 104, 257-261. https://doi. org/10.1034/j.1600-0404.2001.00022.x

Giannaki, C. D., Aristotelous, P., Stefanakis, M., Hadjigeorgiou, G. M., Manconi, M., Leonidou, E., ... Pantzaris, M. (2018). Restless legs syndrome in multiple sclerosis patients: A contributing factor for fatigue, 
impaired functional capacity, and diminished health-related quality of life. Neurological Research, 40(7), 586-592.

Hobart, J., Lamping, D., Fitzpatrick, R., Riazi, A., \& Thompson, A. (2001). The Multiple sclerosis impact scale (MSIS-29): A new patient-based outcome measure. Brain, 124(Pt 5), 962-973.

Hohol, M. J., Orav, E. J., \& Weiner, H. L. (1995). Disease steps in multiple sclerosis: A simple approach to evaluate disease progression. Neurology, 45, 251-255. https://doi.org/10.1212/wnl.45.2.251

Hohol, M. J., Orav, E. J., \& Weiner, H. L. (1999). Disease steps in multiple sclerosis: A longitudinal study comparing disease steps and EDSS to evaluate disease progression. Multiple Sclerosis Journal, 5, 349-354. https://doi.org/10.1177/135245859900500508

Kotterba, S., Neusser, T., Norenberg, C., Bussfeld, P., Glaser, T., Dörner, M., \& Schürks, M. (2018). Sleep quality, daytime sleepiness, fatigue, and quality of life in patients with multiple sclerosis treated with interferon beta-1b: Results from a prospective observational cohort study. BMC Neurology, 18, 123. https://doi.org/10.1186/ s12883-018-1113-5

Krupp, L. B., Larocca, N. G., Muir-Nash, J., \& Steinberg, A. D. (1989). The fatigue severity scale: Application to patients with multiple sclerosis and systemic lupus erythematosus. Archives of Neurology, 46, 1121-1123. https://doi.org/10.1001/archneur.1989.00520 460115022

Learmonth, Y. C., Hubbard, E. A., Mcauley, E., \& Motl, R. W. (2014). Psychometric properties of quality of life and health-related quality of life assessments in people with multiple sclerosis. Quality of Life Research, 23, 2015-2023. https://doi.org/10.1007/ s11136-014-0639-2

Learmonth, Y. C., Motl, R. W., Sandroff, B. M., Pula, J. H., \& Cadavid, D. (2013). Validation of patient determined disease steps (PDDS) scale scores in persons with multiple sclerosis. BMC Neurology, 13, 37. https://doi.org/10.1186/1471-2377-13-37

Lobentanz, I. S., Asenbaum, S., Vass, K., Sauter, C., Klösch, G., Kollegger, H., ... Zeitlhofer, J. (2004). Factors influencing quality of life in multiple sclerosis patients: Disability, depressive mood, fatigue and sleep quality. Acta Neurologica Scandinavica, 110, 6-13. https://doi. org/10.1111/j.1600-0404.2004.00257.x

Lunde, H. M. B., Aae, T. F., Indrevåg, W., Aarseth, J., Bjorvatn, B., Myhr, K. M., \& Bø, L. (2012). Poor sleep in patients with multiple sclerosis. PLoS ONE, 7, e49996. https://doi.org/10.1371/journal.pone.0049996

Manconi, M., Fabbrini, M., Bonanni, E., Filippi, M., Rocca, M., Murri, L., Ferini-Strambi, L. (2007). High prevalence of restless legs syndrome in multiple sclerosis. European Journal of Neurology, 14, 534-539. https://doi.org/10.1111/j.1468-1331.2007.01740.x

Manconi, M., Ferini-Strambi, L., Filippi, M., Bonanni, E., ludice, A., ... Terzano, G. (2008). Multicenter case-control study on restless legs syndrome in multiple sclerosis: The REMS study. Sleep, 31, 944-952.

Marrie, R. A., Zhang, L., Lix, L. M., Graff, L. A., Walker, J. R., Fisk, J. D., ... El-Gabalawy, R. (2018). The validity and reliability of screening measures for depression and anxiety disorders in multiple sclerosis. Multiple Sclerosis and Related Disorders, 20, 9-15. https://doi. org/10.1016/j.msard.2017.12.007
Moreira, N. C. V., Damasceno, R. S., Medeiros, C. A. M., De Bruin, P. F. C., Teixeira, C. A. C., Horta, W. G., \& De Bruin, V. M. S. (2008). Restless leg syndrome, sleep quality and fatigue in multiple sclerosis patients. Brazilian Journal of Medical and Biological Research, 41, 932-937. https ://doi.org/10.1590/s0100-879×2008001000017

Multiple Sclerosis International Federation. (2013) Atlas of MS: Mapping multiple sclerosis around the world. Available at: https://www. msif.org/wp-content/uploads/2014/09/Atlas-of-MS.pdf. Accessed: October 24, 2018

National Institute of Mental Health. (2018) National Institute of Mental Health: Anxiety Disorders. Retrieved on: October 23, 2018. Available at: https://www.nimh.nih.gov/health/topics/anxiety-disorders/ index.shtml

Nourbakhsh, B., Julian, L., \& Waubant, E. (2016). Fatigue and depression predict quality of life in patients with early multiple sclerosis: A longitudinal study. European Journal of Neurology, 23, 1482-1486. https ://doi.org/10.1111/ene.13102

Ohayon, M. M., O'hara, R., \& Vitiello, M. V. (2012). Epidemiology of restless legs syndrome: A synthesis of the literature. Sleep Medicine Reviews, 16, 283-295. https://doi.org/10.1016/j.smrv.2011.05.002

Patriquin, M. A., \& Mathew, S. J. (2017). The neurobiological mechanisms of generalized anxiety disorder and chronic stress. Chronic Stress, 1, [Epub ahead of print]. https://doi.org/10.1177/2470547017703993

Restless Legs Syndrome Foundation. (2018). Medications. Available at: https://www.rls.org. Accessed: May 28, 2018.

Sieminski, M., Losy, J., \& Partinen, M. (2015). Restless legs syndrome in multiple sclerosis. Sleep Medicine Reviews, 22, 15-22. https://doi. org/10.1016/j.smrv.2014.10.002

Tabrizi, F. M., \& Radfar, M. (2015). Fatigue, sleep quality, and disability in relation to quality of life in multiple sclerosis. International Journal of MS Care, 17, 268-274. https://doi.org/10.7224/1537-2073.2014-046

Wallin, M. T., Culpepper, W. J., Campbell, J. D., Nelson, L. M., LangerGould, A., Marrie, R. A., ... Buka, S. L. (2019). The prevalence of MS in the United States: A population-based estimate using health claims data. Neurology, 92, e1029-e1040. https://doi.org/10.1212/ wnl.0000000000007035

Walters, A. S., LeBrocq, C., Dhar, A., Hening, W., Rosen, R., Allen, R. P., ... International Restless Legs Syndrome Study Group. (2003). Validation of the International Restless Legs Syndrome Study Group rating scale for restless legs syndrome. Sleep Medicine, 4, 121-132.

Zigmond, A. S., \& Snaith, R. P. (1983). The hospital anxiety and depression scale. Acta Psychiatrica Scand., 67, 361-370. https://doi. org/10.1111/j.1600-0447.1983.tb09716.x

How to cite this article: Cederberg KLJ, Jeng B, Sasaki JE, Braley TJ, Walters AS, Motl RW. Restless legs syndrome and health-related quality of life in adults with multiple sclerosis. J Sleep Res. 2020;29:e12880. https://doi.org/10.1111/jsr.12880 